\section{CGM LIFE ESERVICES}

Zeit und Kosten sparen -

neue Patienten gewinnen

- Zeitersparnis für Sie und Ihr Team Moderner Service sichert Patientenbindung

Image-Plus für Neupatienten-

page können Ihre Patienten ganz komvom PC, Tablet oder Smartphone Termine in Ihrer Praxis buchen. vollständige Integration der CGM LIFE Patienten buchen Termine in Ihrer Praxis, e eSERVICES, sodass alle Daten immer perund kein klingelndes Telefon hält Ihr fekt synchronisiert sind. Das gilt auch fü Team von der Be- die onlineBefunde und die onlineSprech- The Team von der Betreuung der Pati- stunde zur schnellen Kommunikation mi enten ab: Genau den Patienten. Übrigens: Mit den CGM das ist die Idee If 7 hinter dem AddOn-Modul CGM Ihrer Praxis-Home-

cos effektiv Ihre Selbstzahlerleistungen stegern - gut für Ihren Praxiserfolg.

Mit den CGM LIFE eSERVICES bieten Si Ihren Patienten nicht nur einen innovativen 24/7-Service, der sehr gut in eine modern Lebensgestaltung passt. Intelligente Funktionen fur die Zeit- und Ressourcenplanung optimieren auch den Workflow Ihrer Praxis.

\section{CGM VISODENT 3D AP}

Ihr Beratungs-Einstieg für

beste Patientenbindung $\begin{array}{ll}\text { - } & \text { Perfekte Beratung und Aufklärung } \\ \text { - } & \text { Mehriedene Patienten } \\ & \text { Praxis }\end{array}$

Die Möglichkeiten der zahnärztlichen Behandlung werden immer komplexer. Vieles ist heute machbar, was noch vor einger Zeit undenkbar war - aber es mus immer auch wirtschaftlich tragbar sein. Mit der CGM VISODENT 3D App - kostenlos im iTunes-AppStore erhälttich - können Sie Ihren Patienten in uberzeugenden grafischen Darstellungen die von Ihnen vorgeschlagene Behandlung beispielhaft vorstellen. Die App ist die passende Ergänzung zum CGM Z1-Modul CGM 3DPatientenberater, mit dem Sie die Behandungsschitte exakt anhand der tatsachlirealistisch visualisieren können. Jetzt kostenlos im AppStore herunterladen!

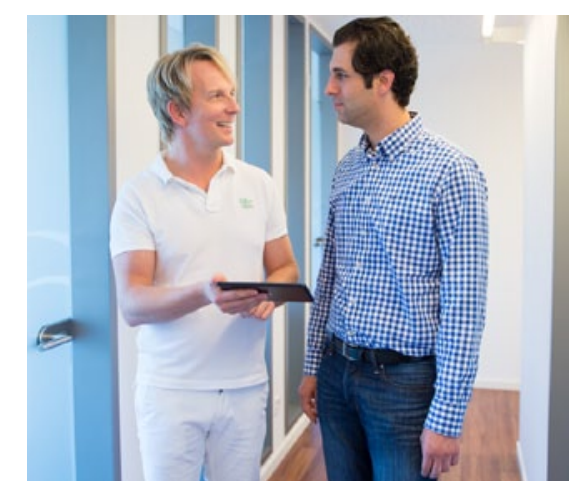

Bilder sagen mehr als Worte - und überzeugen auch viel schneller, gerade wenn es um sehr anspruchsvolle und kostenintenSive Eingrife getht. So geben Sie thren Pati-

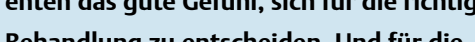
richtige Praxis Das Ergan. Und Z

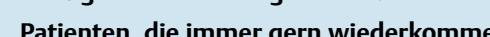
Pier immer gern wiederkommen. fortabel von überall her und zu jeder Zei

Die CGM Dentalsysteme bieten Ihnen die

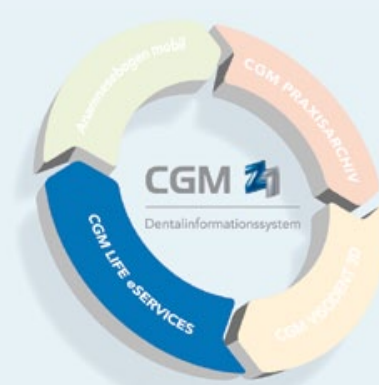

Ihre Praxis der Zukunft: Wirtschaftlich erfolgreich und mit Image-Bestwerten bei den Patienten.
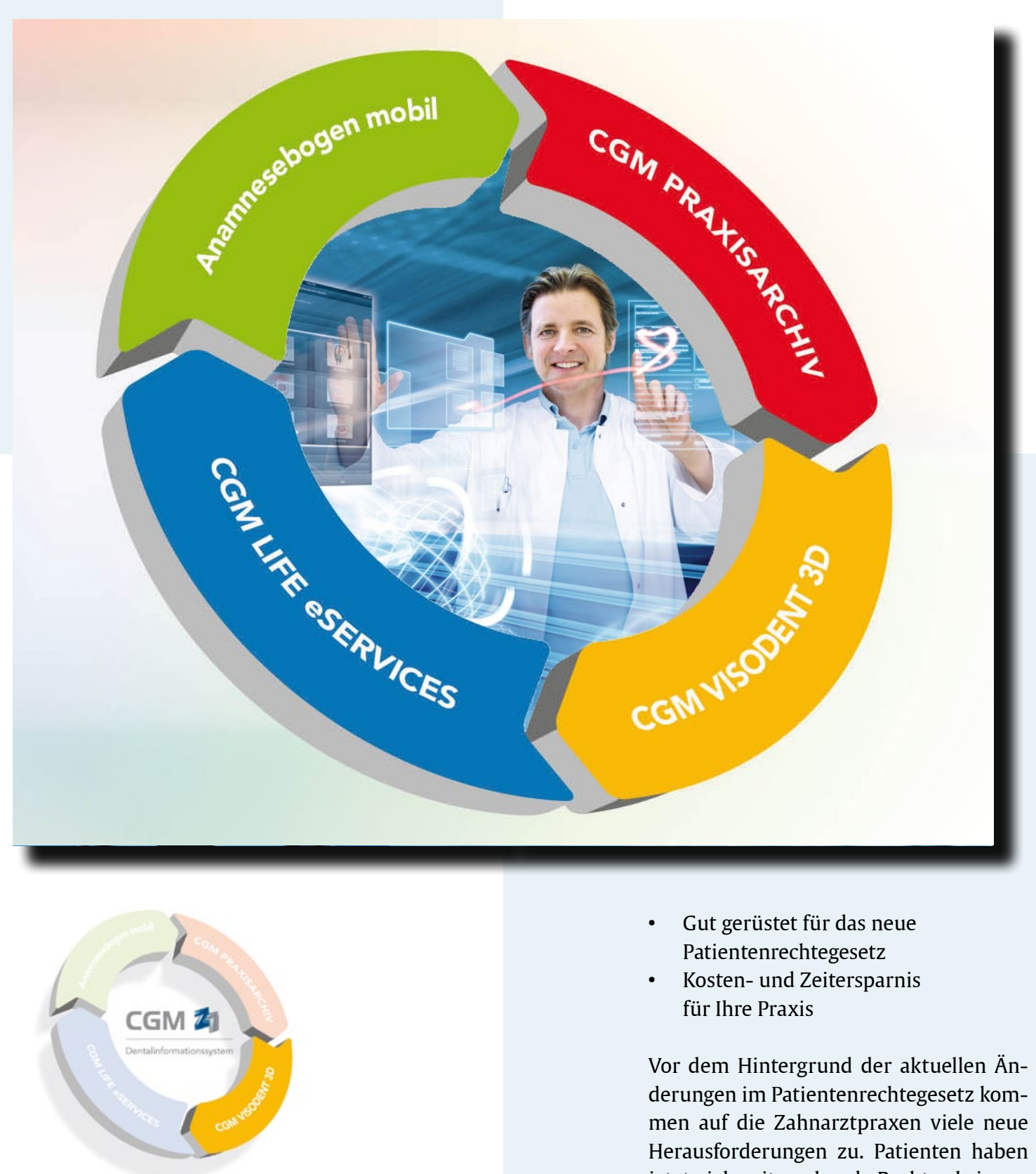

Ihre Praxis der Zukunft: Gut informierte, zufriedene erfolgreiche
- Gut gerüstet für das neue Patientenrechtegesetz Kosten- und Zeitersparnis für Ihre Praxis

Vor dem Hintergrund der aktuellen Änderungen im Patientenrechtegesetz kommen auf die Zahnarztpraxen viele neue jetzt viel weitergehende Rechte als je zuher wh ziehboit und vor allem Vollständigket oberstes Gebot - erst recht, wenn es zu Herausforderungen zu. Patienten haben

- Keine Übertragungsfehler mehr Sicherheit moderne Praxis

So einfach wird das Leben in Ihrer Praxis mit dem Add-On-Modul Anamnesebogen mobil: Statt eines unattraktiven Formu-

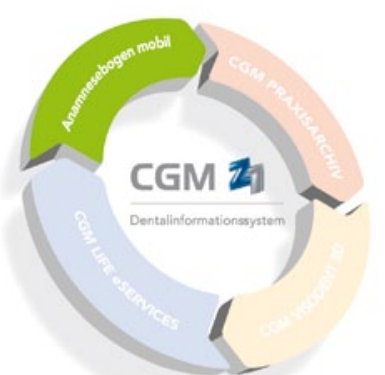

Ihre Praxis der Zukunft: Maximaler Komfort für Ihre Patienten, optimaler Workflow für Ihre Praxis.

Revisionssichere digitale Archivierung Davon haben beide Seiten etwas: Für die

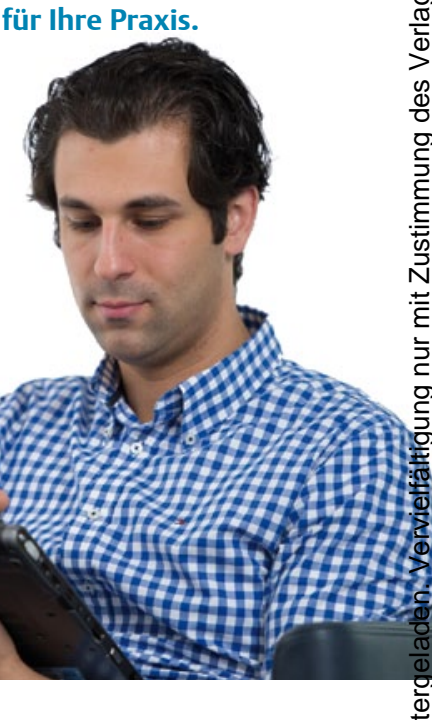

inungsverschiedenheiten mit einem Patienten kommen sollte. Höchste Zeit also, Ihre Patientendaten-Archivierung
und Behandlungsdokumentation auf den neuesten Stand zu bringen

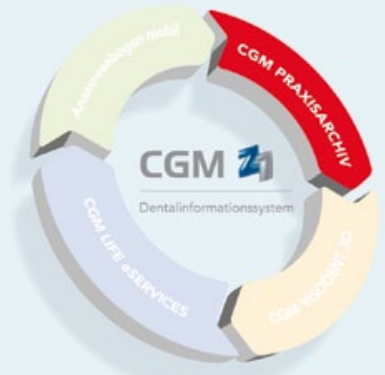

Ihre Praxis der Zukunft: Revisionssichere Datenarchivierung Siegel und REFA bestätigt. Mit freundlicher Unterstützung von
CompuGroup Medical Dentalsysteme,
8)
CGM PRAXISARCHIV

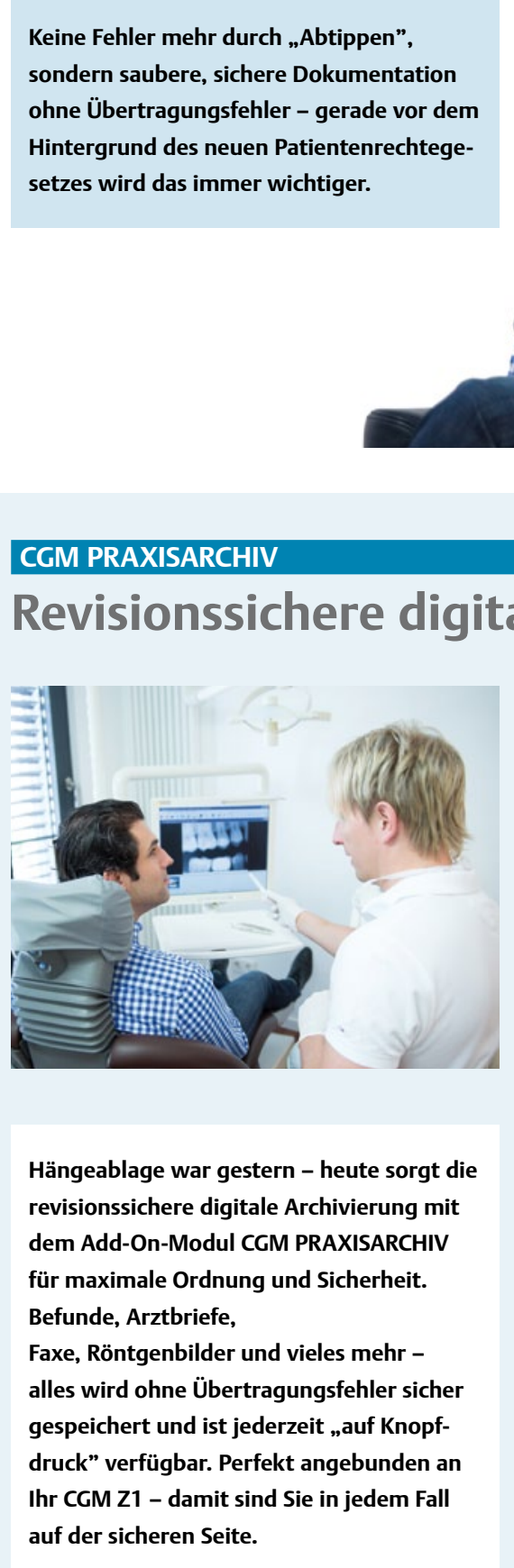

Hängeablage war gestern - heute sorgt die revisionssichere digitale Archivierung mit dem Add-On-Modul CGM PRAXISARCHIV fur maximale Ordnung und Sicherheit. Befunde, Arztbriefe,

Faxe, Rontgenbilder und vieles mehr alles wird ohne Ubertragungsfehler sicher gesuck" verfüggar. Perfekt ang "antoph Ir CGM 71 - dmitsind Sie injedem Fol auf der sicheren Seite. gaben machen können. kaum. Für Ihre Praxis bedeutet das einen wesentlichen Zeit- und Sicherheitsgewinn, denn die Daten können sofort in Ihr übernommen werden. einen Tablet-PC in die Hand, auf dem sie 